\title{
Statistics of white dwarf properties in intermediate polars
}

\author{
Valery F. Suleimanov ${ }^{1,2,3}$ (i), Victor A. Doroshenko ${ }^{1,3}$ and \\ Klaus Werner ${ }^{1}$ \\ ${ }^{1}$ Institut für Astronomie und Astrophysik, Universität Tübingen, \\ Sand 1, D-72076, Tübingen, Germany \\ email: suleimanov@astro.uni-tuebingen.de \\ ${ }^{2}$ Kazan Federal University, Kremlevskaya 18, 420008 Kazan, Russia \\ ${ }^{3}$ Space Research Institute of the Russian Academy of Science, \\ Profsoyuznaya 84/32, 117997 Moscow, Russia
}

\begin{abstract}
Many intermediate polars are hard X-ray sources. The theory of their hard X-ray radiation is well developed and allows us to determine white dwarf masse in this kind of cataclysmic variables. Here we present the results of determination of the masses of 35 white dwarfs in the intermediate polars observed by observatories NuSTAR (10 sources) and Swift/BAT (25 sources). The corresponding mass accrerion rates and the luminosity function were also derived due to accurate distances to the sources well known now after Gaia DR2.
\end{abstract}

Keywords. X-rays: binaries, novae, cataclysmic variables, stars: luminosity function, mass function

\section{Introduction}

A typical Intermediate Polar (IP) is a semi-detached close binary system with a magnetized white dwarf (WD) as a primary (see overview by Warner 2003). The IPs are a subclass of Cataclysmic Variable Stars (CVs). The WD accretes matter from a secondary normal star which overflows its Roche lobe. The accreting matter forms an accretion disc around the WD, which is destroyed by the WD magnetic field at some magnetospheric radius $R_{\mathrm{m}}$. The matter infalls further along magnetic field lines on the WD magnetic poles and forms a strong shock above the WD surface (Aizu 1973; Fabian et al. 1976). Hot, optically thin, post-shock matter heated up to almost the virial temperature settles down on the WD surface. This post-shock region (PSR) is a hard X-ray source, and as a result IPs are numerous Galactic hard X-ray sources on the sky (see e.g. Barlow et al. 2006 and the recent review by Mukai 2017). The maximum PSR temperature and, therefore, the X-ray spectrum hardness depends on the free-fall velocity at the WD surface. The value depends both on the WD mass $M$ and the radius $R$. As the WD radius is related to its mass, it is possible to determine the masses of WDs in IPs using their hard X-ray spectra (Rothschild et al. 1981). This method was used by many authors for WD mass determination using various PSR models and X-ray observations (see, e.g. Cropper et al. 1999, Suleimanov et al. 2005, Brunschweiger et al. 2009, Yuasa et al. 2010, and Shaw et al. 2018). Here we present some new results describing WD properties obtained using a new grid of PSR spectral models, new IP observations performed by observatories NuSTAR (Harrison et al. 2013) and Swift/BAT (Gehrels et al. 2004) and new information about distances to IPs from Gaia Data Release 2 (Gaia collaboration 2018). 


\section{Model and observational data}

We used recently developed PSR models described by Suleimanov et al. (2016). The main features of these models are a quasi-dipole geometry and the finite initial height of the infalling matter. The latter parameter mimics a finite magnetospheric radius $R_{\mathrm{m}}$ and it is potentially important for IPs with small magnetospheres, such as EX Hya (see, e.g. Revnivtsev et al. 2009). The computed grid of PSR spectra is a two-parameter grid with a WD mass $M$ and the relative magnetospheric radius $R_{\mathrm{m}} / R$ as the parameters. This grid is available in XSPEC $\dagger$. The height of the shock could be rather high in low luminous IPs. Therefore, we additionally computed a similar grid with the fixed relative PSR height $H / R=0.25$ and used it for the low luminous IPs studies. The magnetospheric radius of the every IP was found either from the breaking frequency in the power density spectrum (see detailed description of the approach in Suleimanov et al. 2016) or set equal to the corotation radius. This approach allows us to definitely determine the WD mass in the IP using the two-parameter described grid by fitting its hard X-ray spectrum.

The above method was applied to ten IPs observed with NuSTAR observatory, and 25 IPs observed with Swift/BAT observatory, whose spectra are available after publishing of the 105-Month Hard X-ray Survey (Oh et al. 2018). We used only observed spectra at energies above $15-20 \mathrm{keV}$ to avoid the problems with contribution of the reflection and the complex absorption in the softer IP X-ray spectra.

\section{Results}

WD masses and mass accretion rates. Using observed hard X-ray spectra of 35 IPs we determined their WD masses. The distances to 34 of these IPs are now available from Gaia DR2 (Bailer-Jones et al. 2018). The reliable distances allow us to determine the accurate mass accretion rates, $\dot{M}$, in the IPs with the finite $R_{\mathrm{m}}$ values and to evaluate the WD magnetic field strength. All the results are described and presented in detail by Suleimanov et al. (2019). The resulting WD mass distribution and the relation between the IP orbital periods and $\dot{M}$ are shown in Fig. 1. The averaged WD mass obtained $\bar{M}=0.79 \pm 0.16 M_{\odot}$ is close to that obtained by Yuasa et al. $(2010)\left(0.88 \pm 0.25 M_{\odot}\right)$ and Bernardini et al. (2012) $\left(0.86 \pm 0.07 M_{\odot}\right)$ for IPs, and to the averaged WD mass in CVs obtained by Zorotovic et al. (2011) (0.83 $\left.\pm 024 M_{\odot}\right)$. The 105-Month BAT Survey contains information about photon indexes $\Gamma$ for all published spectra. We found a relation between $\Gamma$ and the derived WD masses for all 35 considered IPs, $M / M_{\odot}=1.37( \pm 0.07) /(\Gamma-1.07( \pm 0.08))$, see Fig. 2, left panel.

Luminosity function. The distances to the IPs studied and the time averaged fluxes in the band 14-195 keV presented in 105-Month BAT Catalogue were used for deriving the luminosity function of IPs in the Galaxy. The relation between these two values is presented in Fig. 2, right panel. A line showing a distance corresponding to assumed lower detectability flux in the catalogue used $\left(8 \times 10^{-12} \mathrm{erg} \mathrm{s}^{-1} \mathrm{~cm}^{-2}\right.$; Oh et al. 2018) is also depicted. This line determines the maximum distance up to which IP in some luminosity range can be detectable by the BAT.

The luminosity function was found using a simplified method. We assumed that IPs are uniformly distributed in the Galactic disc with a half thickness 130 pc (Revnivtsev et al. 2008) and the averaged stellar density is constant over the Galactic disc and equals to value in the Sun vicinity, $\rho_{\mathrm{St}} \approx 0.04 M_{\odot} \mathrm{pc}^{-3}$ (McKee et al. 2015). The number density of IPs and their normalized luminosity in the given luminosity range were found using the number of IPs within the luminosity range divided by the Galactic disc volume visible up to the maximum detectable distance for this luminosity range.

$\dagger$ https://heasarc.gsfc.nasa.gov/docs/xanadu/xspec/models/ipolar.html 

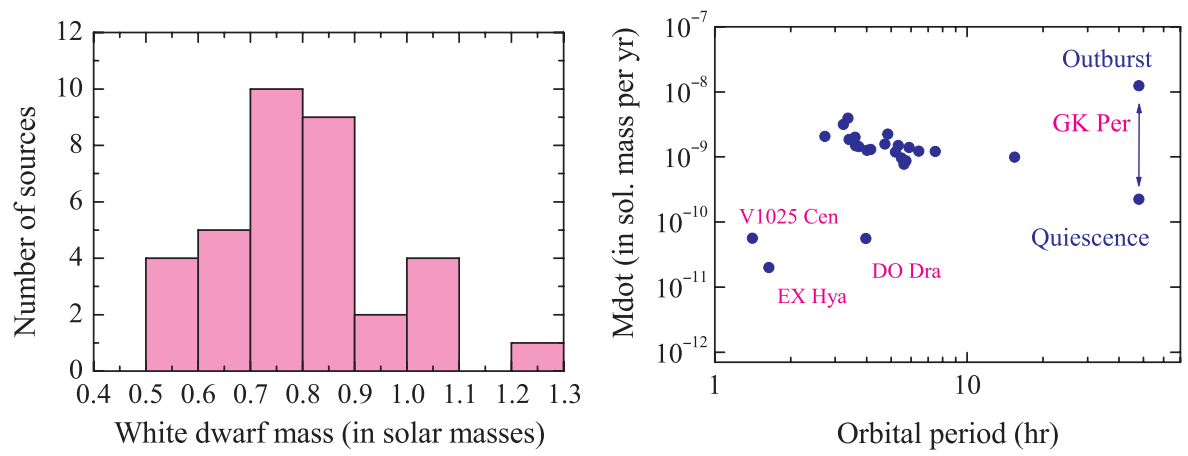

Figure 1. Left panel: Number distribution of WD masses. Right panel: Distribution of derived mass accretion rates in the studied IPs vs. their orbital periods.
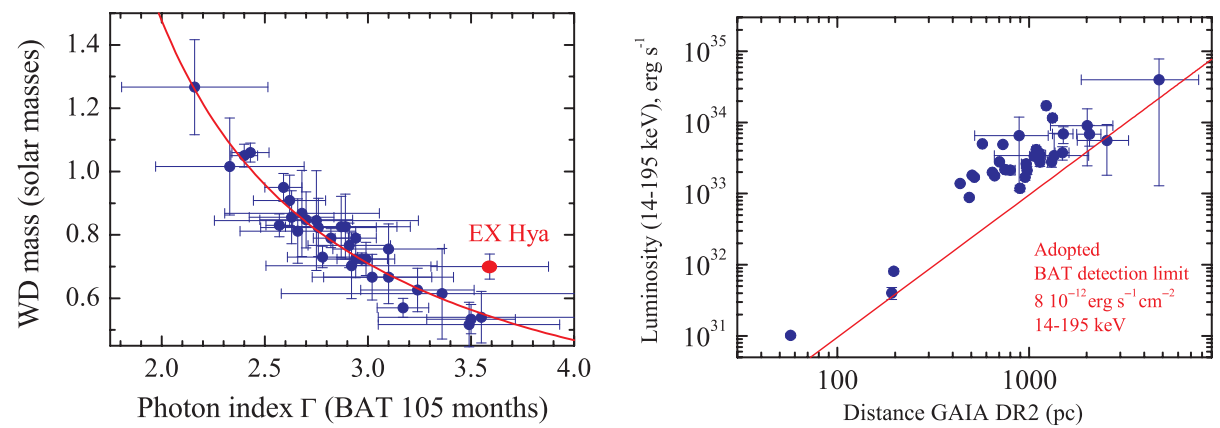

Figure 2. Left panel: Relation between WD masses determined using the fitting of the observed by BAT and NuSTAR spectra with the model spectra and the photon index $\Gamma$ for the same IPs. The best fit of the relation is also shown. EX Hya deviates from the relation due to very small magnetospheric radius. Right panel: Relation between hard X-ray luminosities of the IPs studied in BAT observational band and the distances to them. The adopted lower limit of the detection for used 105-Month BAT catalogue is also shown.
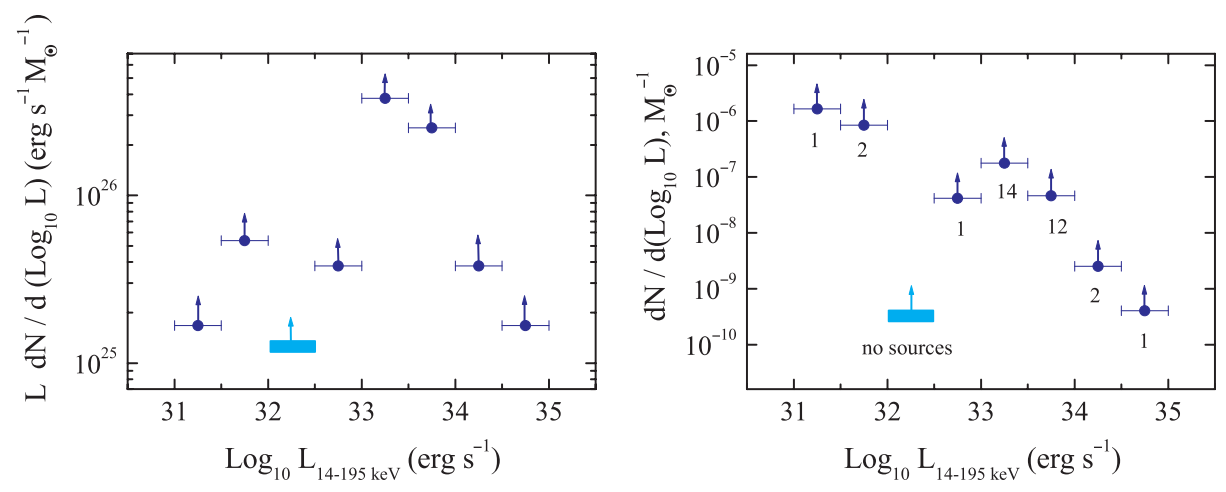

Figure 3. Left panel: Differential luminosity distribution of 14-195 keV emissivity per solar mass of the investigated IPs. Right panel: Luminosity function of the investigated IPs, demonstrating the number of IPs with different luminosities per solar mass at the Solar vicinity. The numbers under the points show the number of IPs in the given luminosity bin.

The results are presented in Fig. 3. The right panel shows the contribution of the given luminosity range to the total luminosity of IPs normalized to one solar mass, whereas the left panel shows the contribution of the IPs in the given luminosity range into the total 
number density of IPs also normalized to the solar mass. Both distributions have a twopike structure reflecting the differences between mass accretion rates in IPs before and after the period gap (see Fig. 1, right panel). The derived luminosity functions are similar to the luminosity functions of CVs detected by INTEGRAL all-sky survey presented in Revnivtsev et al. (2008), see their Figs. 3 and 8.

The averaged number $\rho_{\mathrm{N}} \approx 1.14 \times 10^{-7} \mathrm{pc}^{-3}$ derived from our studies and luminosity $\rho_{\mathrm{L}} \approx 8 \times 10^{26} \mathrm{erg} \mathrm{s}^{-1} M_{\odot}^{-1}$ densities of IPs are close to values obtained by Revnivtsev et al. $(2008), \rho_{\mathrm{N}}=1.5 \pm 0.6 \times 10^{-7} \mathrm{pc}^{-3}$ and $\rho_{\mathrm{L}}=1.3 \pm 0.3 \times 10^{27} \mathrm{erg} \mathrm{s}^{-1} M_{\odot}^{-1}$ correspondingly. The value of the averaged IP number density $\rho_{\mathrm{N}}=1_{-0.5}^{+1} \times 10^{-7} \mathrm{pc}^{-3}$ derived by Pretorius \& Mukai (2014) is also close to our value. We note, however, that the luminosity functions and average densities presented here are low limits only, because the 105-Month BAT catalogue consists of at least in two times more IPs than we used here. More accurate luminosity functions will be presented in the separate paper.

\section{Acknowlegements}

The work was supported by the German Research Foundation(DFG) grant WE 1312/51-1, and the Russian Foundation for BasicResearch grant 18-42-160003 ra (VFS).

\section{References}

Aizu, K. 1973, Progr. Theor. Phys., 49, 1184

Bailer-Jones, C. A. L., Rybizki, J., Fouesneau M., et al. 2018, AJ, 156, 58

Barlow, E. J., Knigge, C., Bird, A. J. J., et al. 2006, MNRAS, 372, 224

Bernardini, F., de Martino, D., Falanga M., et al. 2012, Astr. and Astrophys., 542, A22

Brunschweiger, J., Greiner, J., Ajello, M., \& Osborne, J. 2009, Astr. and Astrophys., 496, 121

Cropper, M., Wu, K., Ramsay, G., \& Kocabiyik, A. 1999, MNRAS, 306, 684

Fabian, A. C., Pringle, J. E., \& Rees, M. J. 1976, MNRAS, 175, 43

Gaia collabotration, 2018, Astr. and Astrophys., 616, A1

Gehrels, N., Chincarini, G., Giommi, P., et al. 2004, ApJ, 611, 1005

Harrison, F. A. et al. 2013, ApJ, 770, 103

McKee, C. F., Parravano, A., \& Hollenbach, D. J. 2015, ApJ, 814, 13

Mukai, K. 2017, PASP, 129, 062001

Oh. K. et al. 2018, ApJS, 235, 4

Pretorius, M. L. \& Mukai, K. 2014, MNRAS, 442, 2580

Revnivtsev, M. et al. 2008, Astr. and Astrophys., 489, 1121

Revnivtsev, M. et al. 2009, A\&SA, 507, 1211

Rothschild, R. E. et al. 1981, ApJ, 250, 723

Shaw, A. W., Heinke, C.O., Mukai, K., et al. 2018, MNRAS, 476, 554

Suleimanov, V., Revnivtsev, M., \& Ritter, H. 2005, Astr. and Astrophys., 435, 191

Suleimanov, V., Doroshenko, V., Ducci, L., et al. 2016, Astr. and Astrophys., 591, A35

Suleimanov, V., Doroshenko, V., \& Werner, K. 2019, MNRAS, 482, 3622

Warner, B. 2003, Cataclysmic Variable Stars, Cambridge Univ. Press, Cambridge, UK

Yuasa, T., Nakazawa, K., Makishima, K., et al. 2010, Astr. and Astrophys., 520, A25

Zorotovic, M., Schreiber, M. R., \& Gänsicke, B. T. 2011, Astr. and Astrophys., 536, A42 